\title{
A Pilot Evaluation of Dialectical Behavioural Therapy in Adolescent Long-Term Inpatient Care
}

\author{
Michael G. McDonell ${ }^{1}$, Jessica Tarantino ${ }^{2}$, Anthony P. Dubose ${ }^{1,4}$, Patricia \\ Matestic $^{6}$, Kristin Steinmetz ${ }^{3}$, Holly Galbreath ${ }^{5}$ \& Jon M. McClellan ${ }^{1,2,3}$
}

${ }^{1}$ Department of Psychiatry and Behavioural Sciences, University of Washington School of Medicine, Box 359911, Harborview Medical Center, $3259^{\text {th }}$ Ave, USA. E-mail: mikemcd@u.washington.edu

${ }^{2}$ Children's Hospital and Regional Medical Center, Seattle, USA

${ }^{3}$ Child Study and Treatment Center, Tacoma, USA

${ }^{4}$ Dialectical Behavioral Therapy Center of Seattle, USA

${ }^{5}$ Clover Park School District, Lakewood, USA

${ }^{6}$ Madigan Army Medical Center, Tacoma, USA

\begin{abstract}
Background: There is a paucity of research investigating psychosocial treatments for youth receiving long-term residential care. Objective: This study describes the implementation and impact of dialectical behavioural therapy (DBT) in a long-term psychiatric hospital located in the United States of America. Method: Changes in overall functioning, number of psychotropic medications prescribed, non-suicidal self-injurious behaviour (NSIB), and locked seclusions were investigated in 106 consecutive unique adolescent patients who received DBT. In addition, a comparison group of historical controls was used to examine the effect of DBT in youth with the highest rates of NSIB. Results: A statistically significant increase in overall functioning, as well as a decrease in number of psychotropic medications and non-suicidal self-injurious behaviour (NSIB) was observed within the DBT group. A decrease in locked seclusions was not observed. Accounting for the effects of age, gender, length of stay, and time, youth who received DBT were less likely to engage in NSIB relative to historical controls. Conclusions: These preliminary data suggest that DBT is beneficial for youth with NSIB in long term inpatient psychiatric care.
\end{abstract}

\section{Key Practitioner Message:}

- DBT is a feasible treatment in long-term inpatient care.

- Relative to a comparison group, DBT appeared to reduce non-suicidal self injurious behaviour in this setting.

- No effect of DBT was observed on the frequency of locked seclusions.

- While maintaining treatment integrity, it was possible to tailor the intensity of DBT to specific groups of patients.

Keywords: Dialectical behaviour therapy, adolescents, inpatient psychiatric care, long term.

Dialectical behavioural therapy (DBT) is an empirically supported treatment developed to reduce suicidal behaviour, non-suicidal self-injurious behaviour (NSIB), and other impulsive behaviours in persons with borderline personality disorder (Linehan, 1993) and has demonstrated reductions in psychiatric hospitalization (Verheul et al., 2003), self-harm (Linehan et al., 1991), and depression (McQuillan et al., 2005).

DBT has been adapted for adolescents with NSIB and emotion dysregulation (Miller, Rathus, \& Linehan, 2007). Two university medical centre based trials utilised quasi-experimental designs to evaluate the relationship between DBT and outcomes in adolescents with histories of NSIB were conducted in the United
States of America (Rathus \& Miller, 2002) and Canada (Katz et al., 2004). Katz and colleagues demonstrated feasibility of DBT in an acute inpatient setting, where DBT was associated with reductions in behavioural incidents during inpatient care, relative to a treatment as usual control group. Miller et al. (2007) found similar outcomes, relative to a comparison group, in an outpatient adolescent sample. A community based pilot study conducted in the United Kingdom of 16 outpatient adolescent females also found positive effects of DBT (James et al., 2008). Based upon these supportive initial data, DBT is currently being adapted for a variety of adolescent samples, some of which maybe quite different in terms of settings and samples that form the 
base of empirical support (e.g. adolescent females with NSIB). Therefore, data evaluating the impact of DBT on outcomes in novel settings and patient populations, such as youth in long-term psychiatric care, is needed. Further, the impact of DBT on outcomes relevant to inpatient care, such as seclusion and functional status, has not been studied.

There is also a paucity of research investigating empirically supported treatments in long-term inpatient care, despite the high costs associated with longterm inpatient hospitalization. In long-term juvenile detention the impact of DBT was assessed among 45 incarcerated adolescent females with psychiatric disorders (Trupin et al., 2002). Relative to historical controls, a significant decrease in behaviour problems over 10-months was observed.

Based upon previous research and in an effort to reduced length of stay, use of seclusion, and NSIB, a DBT adaptation was developed for adolescents in longterm inpatient care located in the USA. In this adaptation, DBT was the core milieu strategy for all patients. Patients were assigned to varying intensities of DBT based on clinical characteristics (e.g. self-harm history, diagnosis). The impact of DBT was evaluated using outcomes that were gathered in the course of clinical treatment (i.e. NSIB, seclusions, number of prescribed psychotropics, functional impairment). The number of psychotropic medications prescribed and functional impairment at admission and discharge were investigated within the DBT sample. Changes in NSIB and locked seclusions were also investigated across time. We hypothesized that DBT would be associated with improved outcomes during the course of hospitalisation. We also hypothesized that DBT would be associated with decreased rates of NSIB and lock seclusions relative to a historical control group who received inpatient care at this facility prior to implementation of DBT.

\section{Method}

\section{Participants}

DBT participants were 106 youth aged 12 to 17 years $(M=15.54, \quad S D=1.20)$ admitted and discharged between 2000 and 2005. Historical control participants were 104 youth aged $12-15$ years $(M=15.3, \mathrm{SD}=1.1)$ admitted and discharged between 1995 and 1999. The majority of the overall sample was female $(n=121$, $58 \%$ ). Participants were consecutive unique admissions. Voluntary and involuntary admissions were included. Involuntary admissions are defined as individuals who were detained under civil commitment laws because they are judged to be a danger to themselves or others and/or are unable to function in the community. Admissions for legal competence restoration were excluded, as the goal of treatment for these individuals was to restore their legal competency to be tried for criminal offences. The Washington State Institutional Review Board provided human subject approval.

\section{Treatment}

DBT included all components of Linehan's model (Linehan, 1993). All staff, including teachers, recreational therapists, nursing staff, and front line clinical staff received DBT training. Developmental modifications were made to treatment techniques and materials. Treatment group participants received either milieu only (milieu DBT), milieu and DBT skills training group (group DBT), or milieu, skills training group, and individual DBT (full DBT). Assignment to DBT intensity was based on clinical judgment, not random assignment. Milieu DBT included chain analysis of problematic behaviour, behavioural interventions, and skills taught individually. DBT therapists were licensed clinicians and trainees in social work, psychology, and psychiatry. A DBT consultation group met regularly and included all group and individual DBT therapists. Youth not enrolled in full DBT were provided with individual, group and family therapies.

The general treatment setting was a single unit at a long term inpatient psychiatric facility (the facility includes 3 age based treatment units) that specialized in the treatment of older adolescents (14-17 year olds). The typical census in this unit was $12-16$ patients at any given time. Staff included psychiatric technicians, who provided behavioural management and direct supervision of patients, nursing staff, an attending psychiatrist, a social worker and an attending psychologist. All participants, including historical controls, were prescribed psychiatric medications as indicated. All patients received recreational therapy, specially designed educational instruction, case management, and other group psychotherapies. Historical controls received individual and family psychotherapies as indicated.

\section{Procedure}

The first author collected medical record data from the records of the 106 DBT participants. Discharge diagnoses were made by attending psychiatrists responsible for patient care and structured interviews were not used. Dependent measures included length of stay (months), discharge placement, and change in number of psychiatric medications and functional status (ChildGlobal Assessment Scale, CGAS, range 0-100) from admission to discharge. Attending child and adolescent psychiatrists who were University of Washington faculty conducted admission and discharge CGAS ratings. CGAS scores, discharge placement, and change in psychiatric medication data were not available for the comparison group, due to the infeasibility of abstracting these data from the medical record. Therefore, comparisons between the DBT and historical comparison group were not possible across these variables. The electronic hospital quality assurance database was used to assess the frequency of locked seclusions for all 210 participants. NSIB were also obtained from the quality assurance databases. However, these data were only recorded beginning in 1997. Therefore, NSIB data were available for only 49 the 104 historical controls.

\section{Data analysis}

Variables were descriptively analysed. Repeated measures ANOVA were used to assess change in continuous outcomes (number of psychiatric medications \& CGAS scores) within the DBT group. Dichotomous outcomes (locked seclusions and NSIB) were investigated across 12 months post-admission using generalised estimating equations utilizing SPSS Statistics version 17.0. 
Generalised estimating equations are a generalized linear model technique that allows investigation of dichotomous correlated data across time.

\section{Results}

Of participants that received DBT, 73\% $(n=77)$ had a history of suicidal ideation or behaviour; $32 \%(n=34)$ were wards of the state, $66 \%(n=70)$ had a history of child protection system involvement, $52 \%(n=55)$ had suffered sexual abuse, and 68\% $(n=72)$ had juvenile justice involvement. At discharge, patients averaged 3 (1.4) Axis I diagnoses, with oppositional defiant (81\%, $n=86)$, conduct disorders $(71 \%, n=75)$, and internalizing disorders (major depression $=52 \%, n=55$; post-traumatic stress disorder $=45 \%, n=45$ ) the most common. At discharge, antipsychotic medications were most frequently prescribed $(51 \%, n=54)$, followed by antidepressants $(42 \%, n=45)$, anti-convulsants $(29 \%$, $n=31)$, anxiolytics $(17 \%, n=18)$, stimulants $(12 \%$, $n=13)$, and lithium $(5 \%, n=5)$.

These variables were descriptively examined across DBT intensities. Since patient characteristics were not independent from the type of DBT prescribed, statistical comparisons were not conducted. Youth receiving full DBT were likely to be female, had high rates of mood disorders, post-traumatic stress disorder, cluster B personality disorder traits, sexual abuse histories, and NSIB. Adolescents who received group DBT were often male and diagnosed with externalising disorders. Those who received milieu DBT were likely to have a psychotic illness or developmental disability. Youth in the historical control group were similar to those receiving DBT in regards to age, gender, and length of stay.

\section{Treatment outcomes}

A statistically significant increase of 14.14 points $(S D=17.52)$ in CGAS scores from admission to discharge was observed, $F(1,97)=154.86, p<.001$, for youth who received DBT. On average, DBT participants were prescribed 2.4 psychotropic medications $(S D=1.7)$ at admission and $1.1(S D=1.4)$ at discharge, a statistically significant reduction, $F(1,97)=55.89$, $p<.001$. Forty-three percent $(n=49)$ were discharged to their parents, $22 \%(n=25)$ to foster care, $18 \%$ $(n=20)$ to a group home, and $2 \%(n=2)$ to long-term inpatient treatment.

The relationships between DBT and locked seclusion and NSIB were investigated for the first year of treatment using generalised estimating equations. Accounting for gender, age, and length of stay, there was a significant effect of time on NSIB, wald $\chi^{2}$ $(3)=21.1, p<0.001$. The effect of time on locked seclusion was not significant.

Rates of NSIB in youth who received DBT were compared to historical controls. NSIB was infrequent in the overall sample. Fifty-one percent $(n=79)$ had no NSIB, $21 \%(n=33)$ had 1 or 2 NSIB, and $28 \%(n=43)$ had 3 or more NSIB during their treatment. For this analysis, we focused on youth with 3 or more recorded episodes of NSIB. Accounting for gender, age, length of stay, and the effect of time, youth who received DBT $(M=0.59$, $C I=0.49-0.69)$ had significantly lower rates of NSIB across 12 months of hospitalisation, compared to his- torical controls $(M=0.75, \quad C I=0.64-0.86)$, wald $\chi^{2}$ $(1)=4.1, p<.05$.

\section{Discussion}

In our long-term psychiatric inpatient setting, those who received DBT experienced statistically significant improvements across time in global functioning, as well as reductions in prescribed psychotropic medications. Data for these outcomes was not available for comparison group youth; therefore, changes in these variables cannot be directly attributed to DBT. In comparison to historical controls, exposure to DBT was associated with a greater reduction in NSIB, in those youth with the most severe histories of self-injurious behaviours.

This was not a randomised trial. Changes in outcomes cannot be attributed solely to the effects of DBT. However, these data support for use of this model in adolescents in long-term inpatient care, particularly patients with high rates of NSIB. Randomised clinical trials of DBT in this setting are needed to establish its efficacy for this population.

One unique characteristic of this DBT model was the three levels of DBT intensity. This allowed for tailoring of the DBT program to the needs of each patient. Those assigned to treatment groups represented distinct clinical populations. Youths who received full DBT were similar to adults for whom the intervention was designed and to adolescents for whom the intervention has been piloted (James et al., 2008; Katz et al., 2004; Linehan, 1993; Rathus \& Miller, 2002).

Teens who received group DBT were likely to be males with externalising disorders. Group DBT was likely to benefit these youth, as they may have interpersonal skills and emotion regulation difficulties that manifest as externalising behaviours, but do not have persistent NSIB that would require full DBT. Those assigned to milieu DBT typically suffered from psychotic or pervasive developmental disorders. While contingency management principals of DBT provided a foundation for milieu management for these youth, the more complex and cognitive based group and individual components of DBT were less appropriate.

Other study limitations include lack of randomisation to treatment, retrospective data collection, and no measure of DBT adherence. In addition, results may have been influenced by other efforts to respond to reduce length of stay, NSIB, and seclusion and restraint. The implementation of DBT was only one of many quality improvement efforts and staff trainings that occurred during this time period.

This report is one of few that describe outcomes associated with evidence based interventions adapted for adolescent inpatients (Case et al., 2007; Greene, Albon, \& Martin, 2006). Adolescents in long-term care represent a small, but important, group of youth, as they are served by multiple publicly funded systems of care. Therefore, implementation and evaluation of empirically supported treatments for youth in this setting is important in order to improve symptomatic and functional outcomes, as well as prevent long-term disability.

Hospitals and community programs may be hesitant to adopt and adapt empirically supported treatments. 
In our setting, the innovative adaptation of DBT formed the core treatment for youth and was associated with positive therapeutic outcomes. This study demonstrates how treatments, such as DBT, can be adapted and evaluated utilising existing means in real world settings. Future research should focus on evaluating the efficacy and/or effectiveness trails of DBT in this and similar settings. Research that investigates issues related to dissemination, such as DBT model adherence, impact of DBT on staff outcomes, and cost savings are also important.

\section{References}

Case, B.G., Olfson, M., Marcus, S.C., \& Seigel, C. (2007). Trends in the inpatient mental health treatment of children and adolescents in US community hospitals between 1990 and 2000. Archives of General Psychiatry, 64, 89-96.

Greene, R.W., Albon, J.S., \& Martin, A. (2006). Use of collaborative problem solving to reduce seclusion and restraint in child and adolescent inpatient units. Psychiatric Services, 57, 610-612.

James, A.C., Taylor, A., Winmill, L., \& Alfoadari, K. (2008). A preliminary community study of dialectical behaviour threapy (DBT) with adolescent females demonstrating presistent, deliberate self-harm (DSH). Child and Adolescent Mental Health, 13, 148-152.

Katz, L. Y., Cox, B. J., Gunasekara, S., \& Miller, A. (2004). Feasibility of dialectical behavior therapy for suicidal ado- lescent inpatients. Journal of the American Academy of Child and Adolescent Psychiatry, 43, 276-282.

Linehan, M. M. (1993). Cognitive-behavioural treatment of borderline personality disorder. New York: Guilford Press.

Linehan, M. M., Armstrong, H. E., Suarez, A., Allmon, D., \& Heard, H. L. (1991). Cognitive-behavioural treatment of chronically parasuicidal borderline patients. Archives of General Psychiatry, 48, 1060-1064.

McQuillan, A., Nicastro, R., Guenot, F., Girard, M., Lissner, C., $\&$ Ferrero, F. (2005). Intensive dialectical behavior therapy for outpatients with borderline personality disorder who are in crisis. Psychiatric Services, 56, 193-197.

Miller, A.L., Rathus, J.H, \& Linehan, M.M. (2007). Dialectical behavior therapy with suicidal adolescents. New York: Guilford Press.

Rathus, J. H., \& Miller, A. L. (2002). Dialectic behavior therapy adapted for suicidal adolescents. Suicide and Life Threatening Behavior, 32, 146-158.

Trupin, E., Stewart, D.G., Beach, B., \& Boesky, L. (2002). Effectiveness of a dialectical behaviour therapy program for incarcerated female juvenile offenders. Child and Adolescent Mental Health, 7, 121-127.

Verheul, R., Van Den Boxch, L. M. C., Koeter, M. W. J., De Ridder, M.A., Stijnen, T., \& Van Den Brink, W. (2003). Dialectical behaviour therapy for women with borderline personality disorder: 12-month, randomised clinical trial in the Netherlands. British Journal of Psychiatry, 182, 135140 . 